\title{
Stability Analysis for Smart Homes Energy Management System with Delay Consideration
}

\author{
Saher Umer, Yasuo Tan, and Azman Osman Lim
}

\begin{abstract}
There is a tendency to consume electrical energy simultaneously by home appliances (HAs) leading to frequent demand peaks. Nowadays the number of (HAs) in modern residences has significantly increased as compared to the past. If the power demand is not met with the limited available power supply, this could lead to major power blackout. Our smart power management system consists of smart electric sensor (SES) and power provisioning controller (PPC). The PPC and SES are used to control and maintain stability with complete power waveform behavior examination. In this paper, we focus on achieving optimal system model for smart homes to reduce the risks of power blackout by applying two different criteria of power assignment for the HAs. A comparative study has been conducted which show the drastic change in the system behavior by changing the power assignment criteria. We also carry out the stability analysis for the proposed system model of smart homes. The stability analysis verifies that the system stability is dependent on some time delay. Simulation results also help us to observe critical system behaviors such as stability and overshoot of energy waveform.
\end{abstract}

Index Terms-Power provisioning controller, smart electric sensor, maximum consuming power, home appliance power derivation, power supply/demand.

\section{INTRODUCTION}

A system of smart homes refers to a residence that is equipped with computing and information technology, which responds to the needs of the occupants and provides comfort, convenience, security and entertainment [1]. Smart homes expect to control numerous diverse systems in a household domain. Air-conditioning, audio/video, heating, lighting, security and health care, such systems could be found in smart homes [2]. It is already convinced that the number of home appliances (HAs) in smart homes has significantly increased compared to the past. The high power required by HAs makes our homes one of the most critical areas for the impact of power consumption. The real time monitoring of power consumption by HAs is important for future intelligent home where intelligent home is dedicated to the seamless infusion of technology with day to day living to create a lifestyle unique for each individual [3]. As the residential areas are one of the major power consumers today, thus reducing power in home would contribute greatly to the environment. As more and more HAs are introduced with attractive features, the power consumption at home tends to grow and leads to increase the risk of losing stability of the whole power system [4]. In this way, we have to face the

Manuscript received September 30, 2013; revised December 23, 2013.

S. Umer, Y. Tan and A. O. Lim are with Japan Advanced institute of Science and Technology (JAIST). 1-1 Asahidai, Nomi City, Ishikawa, Japan (e-mail: saher, ytan, aolim @ jaist.ac.jp). worst situation of energy shortage. The balancing of energy use is a challenging fundamental issue for the efficient behavior of an electrical system. As a result, we need smart power management system which is responsible for monitoring and operating HAs with limited power supply [5].

A smart power management system for smart homes is a part of the smart grid on the consumption side. It is responsible for collecting data from HAs using sensors, and then optimizing power supply by using the information from sensors [6]. A conventional power management system focused only on power consumption monitoring and standby power reduction strategies [7]. With the increasing power demand by HAs for intelligent and personalized services, smart power management system is required in smart homes to support smart devices. Furthermore, system of smart homes exploits various sensors (e.g. smart meters or smart electric sensors) to improve overall energy efficiency and resident's satisfaction. Large scale usage of sensors with smart appliances leads to a great rise of communication over wired and wireless links, which also requires an intelligent controller, which we will refer to as power provisioning controller $(P P C)$ for managing real time communication. The efficiency, reliability and stability of the smart power management system are expected to be significantly improved with the help of smart electric sensor (SES). It introduced a paradigm shift in the analysis of power system. A SES is used to connect individual HA to the power management system (see Fig. 1).

To reduce maximum consuming power, first it is important to know how much power is consumed. Therefore, power consumption monitoring is needed. The real time monitoring of instantaneous consuming power of the HAs can be successfully achieved by SES. Second, it is also necessary to manage the HAs to apply power reduction strategies with the help of the proposed PPC. To deal with these issues, we propose a simple and effective smart power management system for smart homes consists of SES, PPC and HAs.

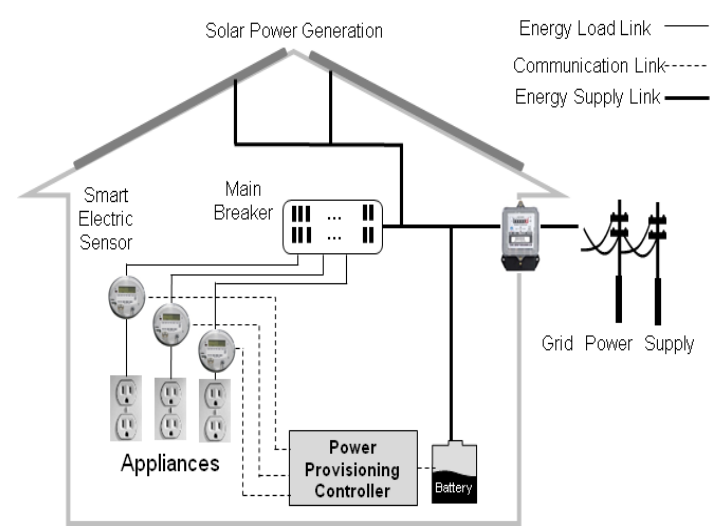

Fig. 1. A sustainable energy house with source and loads. 
In particular, we focus on achieving optimal system model for power management system. We apply two different suggested power assignment criteria to distribute the total available power among HAs. A comparative study has been conducted which shows the change in HA behavior by changing suggested power assignment criteria. The stability theorem for proposed power management system is also presented which shows that the entire system stability is dependent on communication time delay. In the overall scenario, the SES measures the real time instantaneous consuming power levels of HAs and sends this information to PPC. Based on this information, PPC computes the suggested power level for the HAs. The presence of time delay raises the question of stability of the proposed system. The dynamics of the behavior of HAs changes with the change in value of system parameters and introduces critical system events like stable/unstable and overshoot/non-overshoot. At last, simulation results are discussed for the better understanding of the proposed system. In our proposed system design for smart homes, there are two main contributions (i) information delay and (ii) comparison between two suggested power assignment criteria for HAs. These contributions affect critical system power behavior, stable/unstable or in other words overshoot/non-overshoot. Therefore, we need to choose the system delay carefully so that the system would be stable with no overshoot, which is discussed in Section IV.

The rest of the paper is organized as follows. Related work that is associated with this paper is summarized in Section II. In Section III, proposed system model is proposed with its novelty and key features. In Section IV, simulation results are discussed in detail. Section V and Section VI are related with the stability analysis of proposed system and numerical analysis of stability theorem respectively. Finally, we conclude our research with future works in Section VII.

\section{RELATED WORK}

The stability of actual consuming power level is primary research area that needs to be solved. The future smart homes should be dynamic and self-motivated with continual real time communication between HAs, PPC and SESs. Embedding intelligence in the form of energy management system in smart homes to meet the challenges of improved reliability, security, efficiency and system dynamics is a challenging task. The next generation power grid, smart grid, is a dynamic structure with continual real-time two way communication between the producers and consumers. According to the overall trends, the smart grid is an interdisciplinary technology that incorporates the fixed centralized and one-way communication organized power grid with latest developments and novelties. The essential task of electrical power network management systems is to keep the balance between power supply and demand.

Electricity is the basic need for daily life and its failure will probably paralyze the individual household or even the whole city depending on the scale of the failure. Severe misfortunes by large scale power failure have been recognized and many efforts have been done for preventing such a failure [8]-[10]. For controlling and managing HAs, a number of energy management systems were proposed and developed. In [11], the author proposed a method to display power consumption information to the user explaining its overall goals that must be fulfilled. It also provides guidelines for the system development to reduce power consumption. In [12] a research study showed that the feedback on power consumption to power users is effective to reduce total power usage. Pipattanasomporn et al. [13] proposed in-home appliance priority and customer comfort level settings. Gill et al. [14] proposed ZigBee based home automation system, composed of home network devices and an intelligent home gateway. Facchinetti et al [15] presented a method for scheduling to balance the power usage.

The maximum power consumption of the HAs in the user's residence should not exceed a certain limit. In [16]-[18], several approaches were provided and compared to reduce peak power consumption. In these existing studies, there is a lack of power assignment analysis among HAs and effects on HA behavior. In [19]-[21], communication between HAs and controller is held through message exchange but there is lack of consideration of communication delay in message exchange in the overall system scenario. This is our other important contribution and was found to be main factor of the system stability. Kato et al. [22] developed the energy on demand system for single commercial utility power source and proposed demand negotiation algorithm for power sharing scheme to control overall power consumption. The detailed analysis of energy waveform is omitted which is one of the novelties of this paper.

\section{Proposed System Model}

Main breaker of the home is the breaker that the feeder wire associates to. Main breaker is designed to trip if the amperage rating exceeds the value labeled on the breaker. The main breaker is further divided into sub-breakers. Sub-breakers are used to extend feed for multiple branch circuits in whole residential area. From here, the sub-breaker connects to outlets, lighting and other loads via individual circuit breakers. In this paper, we assume that HA is a smart device which changes its power consumption level upon receiving the suggested power from controller.

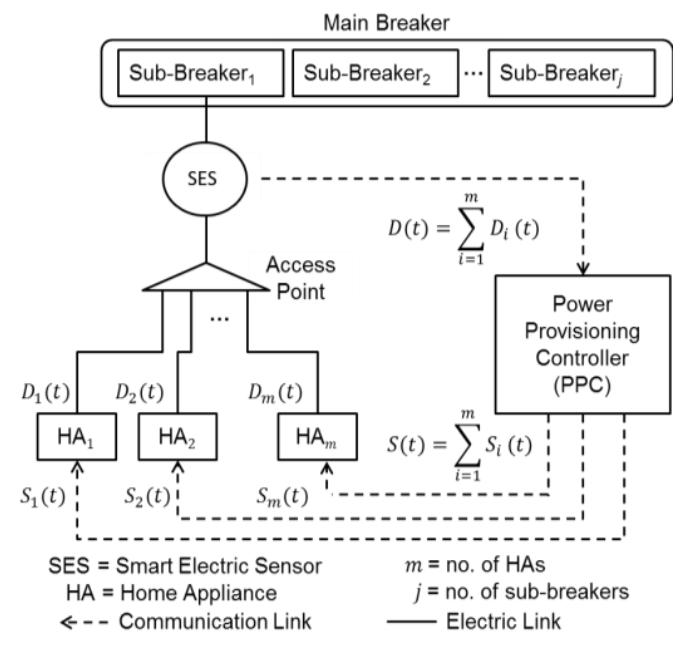

Fig. 2. System model

The proposed system model for smart power management system consists of $m$ HAs $M=\left\{H A_{1}, H A_{2}, \ldots, H A_{m}\right\} \in m$, as depicted in Fig.2. The use of SES helps in measuring the 
instantaneous electric current consumption level of HAs and sends the total $D(t)$ to PPC, which can be calculated as,

$$
D(t)=\sum_{i=1}^{m} D_{i}(t)
$$

where $D_{i}(t)$ is the actual consumed electric current level of appliance $i$ at time $t$. Then the function of PPC is to compute suggested electric current for each HA called $S(t)$. Upon receiving a message from $P P C$, each $\mathrm{HA}$ requires to decide its own operating electric current by considering its own maximum electric current suggested by PPC. We assume that a delay $(\tau)$ is the total time of sending information from the SES to PPC, the processing time at the PPC and the sending information from the PPC to the corresponding HA. In our proposed modeling scheme, the total electric current for all HAs is denoted as $P$, thus remaining electric current power $R(t)$ can be represented as:

$$
R(t)=P-D(t)=P-\sum_{i=1}^{m} D_{i}(t)
$$

We also assume that each HA has its own maximum electric current level, $D_{i}^{\max }$ and it behaves according to the first order state equation given by

$$
\dot{D}_{i}(t)=-a_{i} \cdot D_{i}(t)+b_{i} \cdot u
$$

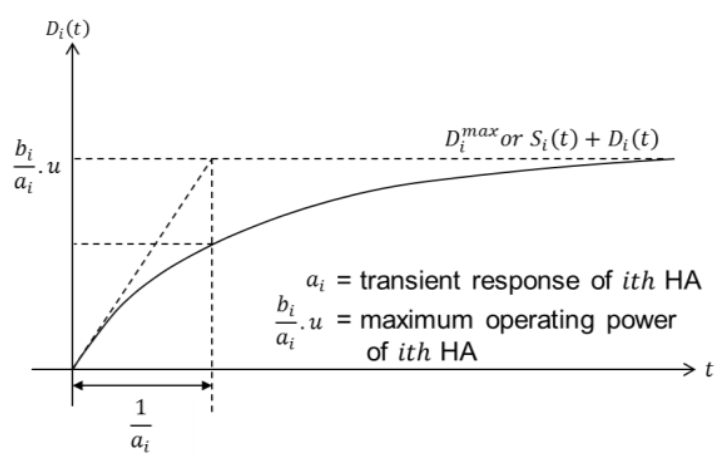

Fig. 3. Home appliance electric current consumption derivation.

where,

$$
b_{i} \cdot u=\left\{\begin{array}{cl}
a_{i} \cdot D_{i}^{\max } & \text { if } S_{i}(t)+D_{i}(t) \geq D_{i}^{\max } \\
a_{i} \cdot\left(S_{i}(t)+D_{i}(t)\right) & \text { if } S_{i}(t)+D_{i}(t)<D_{i}^{\max }
\end{array}\right.
$$

Note that in our first order state equation model, $b_{i} \cdot u / a_{i}$ is the final steady state value of the appliance $i$ and $1 / a_{i}$ is the time constant, i.e. the time required for the electric current of a HA to reach $63 \%$ of the final steady state value (see figure 3 ). In our model $S_{i}(t)+D_{i}(t)$ is considered as an available electric current assigned to each HA, where $S_{i}(t)$ is the delayed version of remaining electric current.

In this case, the ith HA will implement its own operating power at the time $(t-\tau)$. Then, we can write it in the form as:

$$
\dot{D}_{i}(t)=-a_{i} \cdot D_{i}(t)+a_{i} \cdot D_{i}(t-\tau)
$$

For all HAs, the system equation (5) can be represented in matrix notation as:

$$
\begin{gathered}
{\left[\begin{array}{c}
\dot{D}_{1}(t) \\
\vdots \\
\dot{D}_{m}(t)
\end{array}\right]=\left[\begin{array}{ccc}
-a_{1} & \ldots & 0 \\
\vdots & \ddots & \vdots \\
0 & \ldots & -a_{m}
\end{array}\right]\left[\begin{array}{c}
D_{1}(t) \\
\vdots \\
D_{m}(t)
\end{array}\right]} \\
+\left[\begin{array}{ccc}
-a_{1} / l & \ldots & 0 \\
\vdots & \ddots & \vdots \\
0 & \ldots & -a_{m} / l
\end{array}\right]\left[\begin{array}{c}
D_{1}(t-\tau) \\
\vdots \\
D_{m}(t-\tau)
\end{array}\right]+\left[\begin{array}{c}
a_{1} / l \\
\vdots \\
a_{m} / l
\end{array}\right] R
\end{gathered}
$$

Due to message transmission delay and the processing time at PPC, each HA will encounter a delay $(\tau)$. At the point of time $t$, each HA receives its suggested power level $S_{i}(t)$, a delayed version of the remaining electric current that can be calculated by two different electric current assignment criteria; Equal Based Electric Current (EBEC) assignment criteria and Ratio Based Electric Current (RBEC) assignment criteria.

\section{A. EBEC Assignment Criteria}

In this criterion, PPC calculates the remaining electric current $R(t-\tau)$ for the HAs and then divides the remaining electric current by $l$ and sends this information i.e. $S_{i}(t)$ to the HAs where $l$ is the designer's parameter, also called divider of the remaining electric current.

$$
S_{i}(t)=\frac{R(t-\tau)}{l}
$$

\section{B. RBEC Assignment Criteria}

In this criterion, PPC assigns the remaining electric current among HAs according to the following equation.

$$
\begin{gathered}
S_{i}(t)=\frac{r_{i}(t-\tau)}{\sum_{i=1}^{m} r_{i}(t-\tau)} \cdot R(t-\tau) \\
r_{i}(t-\tau)=D_{i}^{\max }-D_{i}(t-\tau)
\end{gathered}
$$

PPC calculates the remaining electric current $R(t-\tau)$ for the HAs and also individual remaining electric current of each HA, $r_{i}(t-\tau)$ that HA needs to reach its maximum consuming electric current level as in (9).

\section{Simulation RESUlts}

For simulation of our proposed system, each appliance is associated with $a_{i}$ and $D_{i}^{\max }$ system parameters which decide the response speed and maximum consuming electric current level of the HA. The information of suggested electric current level is fed back to each HA with time delay $(\tau=1.0)$ from PPC. In the simulation execution with EBEC assignment criteria, we assume $l=3$, the suggested value that can achieve system stability from our previous study [23].

We consider three HAs attached with one SES for clarity of explanation. The total electric current available for all HAs is $P=20 \mathrm{~A}$. The total simulation execution time is 10 seconds. We conduct a computer simulation to analyze our proposed system behavior and summarize our simulation results in given table 1 . The values used for $a_{i}$ and $D_{i}^{\max }$ parameters are shown in the table for each simulation execution. All three HAs are assumed to be turned ON at the same time having same system parameters from the 
beginning of the simulation.

From Table I, the 1st row of RBEC and EBEC have the same behavior. All three HAs share the available electric current equally (see Fig. 4) with same values of system parameters $a_{i}$ and $D_{i}^{\max }$. In these cases, the system show stable behavior without any overshoot. Obviously, the total available electric current is not sufficient for the HAs to reach to its maximum required electric current level. Accordingly, the HAs will saturate at a maximum electric current lower than its required maximum. This is the total electric current distributed evenly among HAs.

The 2nd row of RBEC is similar to the above situation. Now the HAs have different $D_{i}^{\max }$ but the same $a_{i}$. Still the suggested electric current level is less than the required maximum electric current level of each HA (see Fig. 5). In this case again there is no overshoot.

Only the 3rd and 4th rows of RBEC assignment criteria encounter overshoot. The $3 \mathrm{rd}$ has same $D_{i}^{\max }$ but different $a_{i}$ while the latter is vice versa. The 4 th row of RBEC behavior can be seen in Fig. 6, the other one was omitted for space considerations.

In the 2nd row of EBEC, (like 2nd row of RBEC) the HAs have different $D_{i}^{\max }$ and the same $a_{i}$. The two HAs with smaller $D_{i}^{\max }$ reaches to their maximum required consuming electric current level (see Fig. 7). The remaining HA saturates at a level lower than its required maximum.

\begin{tabular}{|c|c|c|c|c|}
\hline $\begin{array}{c}\text { Electric } \\
\text { Current } \\
\text { Assignment } \\
\text { Criteria }\end{array}$ & $a_{i}$ & $D_{i}^{\max }$ & $\begin{array}{c}\text { Overshoot/ } \\
\text { or not? }\end{array}$ & $\begin{array}{l}\text { Stable } \\
\text { Time }\end{array}$ \\
\hline RBEC & $\begin{array}{l}a_{1}=10 \\
a_{2}=10 \\
a_{3}=10\end{array}$ & $\begin{array}{l}D_{1}^{\max }=10 \\
D_{2}^{\max }=10 \\
D_{3}^{\max }=10\end{array}$ & No & $\begin{array}{l}\text { Stable all } \\
\text { the time }\end{array}$ \\
\hline RBEC & $\begin{array}{l}a_{1}=10 \\
a_{2}=10 \\
a_{3}=10\end{array}$ & $\begin{array}{c}D_{1}^{\max }=12 \\
D_{2}^{\max }=7 \\
D_{3}^{\max }=5\end{array}$ & No & $\begin{array}{l}\text { Stable all } \\
\text { the time }\end{array}$ \\
\hline RBEC & $\begin{array}{c}a_{1}=30 \\
a_{2}=10 \\
a_{3}=3\end{array}$ & $\begin{array}{l}D_{1}^{\max }=10 \\
D_{2}^{\max }=10 \\
D_{3}^{\max }=10\end{array}$ & Yes & $\begin{array}{c}\text { Stable after } \\
t=2.13 \mathrm{~s}\end{array}$ \\
\hline RBEC & $\begin{array}{c}a_{1}=30 \\
a_{2}=10 \\
a_{3}=3\end{array}$ & $\begin{array}{c}D_{1}^{\max }=12 \\
D_{2}^{\max }=7 \\
D_{3}^{\max }=5\end{array}$ & Yes & $\begin{array}{c}\text { Stable after } \\
t=1.49 \mathrm{~s}\end{array}$ \\
\hline EBEC & $\begin{array}{l}a_{1}=10 \\
a_{2}=10 \\
a_{3}=10\end{array}$ & $\begin{array}{l}D_{1}^{\max }=10 \\
D_{2}^{\max }=10 \\
D_{3}^{\max }=10\end{array}$ & No & $\begin{array}{l}\text { Stable all } \\
\text { the time }\end{array}$ \\
\hline EBEC & $\begin{array}{l}a_{1}=10 \\
a_{2}=10 \\
a_{3}=10\end{array}$ & $\begin{array}{c}D_{1}^{\max }=12 \\
D_{2}^{\max }=7 \\
D_{3}^{\max }=5\end{array}$ & No & $\begin{array}{l}\text { Stable all } \\
\text { the time }\end{array}$ \\
\hline EBEC & $\begin{array}{c}a_{1}=30 \\
a_{2}=10 \\
a_{3}=3\end{array}$ & $\begin{array}{l}D_{1}^{\max }=10 \\
D_{2}^{\max }=10 \\
D_{3}^{\max }=10\end{array}$ & Yes & $\begin{array}{c}\text { Stable at } \\
t=2.08 \mathrm{~s}\end{array}$ \\
\hline EBEC & $\begin{array}{c}a_{1}=30 \\
a_{2}=10 \\
a_{3}=3\end{array}$ & $\begin{array}{c}D_{1}^{\max }=12 \\
D_{2}^{\max }=7 \\
D_{3}^{\max }=5\end{array}$ & Yes & $\begin{array}{l}\text { Stable at } \\
t=2.00 \mathrm{~s}\end{array}$ \\
\hline
\end{tabular}

Finally both the 3rd and 4th rows of EBEC encounter overshoot. Both have different $a_{i}$, the former has same $D_{i}^{\max }$ while the latter has different values. As with the 3rd and 4th rows of RBEC, only the 4th row of EBEC behavior shown in Fig. 8.

We can conclude that if the time constant for each HA is same, we can keep the maximum electric current limit.
However, if the time constant for each HA is different the proposed scheme cannot guarantee maximum electric current limit.

We also conclude that with same $D_{i}^{\max }$ or different $D_{i}^{\max }$, if the system parameter $a_{i}$ is the same, then the system will be stable in either of the proposed assignment criteria.

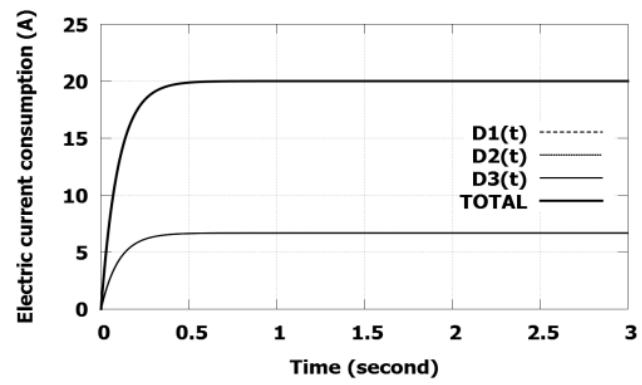

Fig. 4. Electric current consumption behavior of RBEC and EBEC criteria with $a_{i}=10$ and $D_{i}^{\max }=10$ (for HA $i=1,2,3$ ).

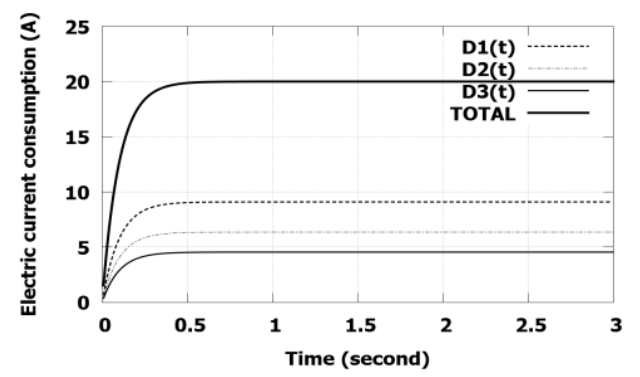

Fig. 5. Electric current consumption behavior of RBEC criteria with $a_{i}=10$ and $D_{i}^{\max }=12,7,5$ (for HA $i=1,2,3$ ).

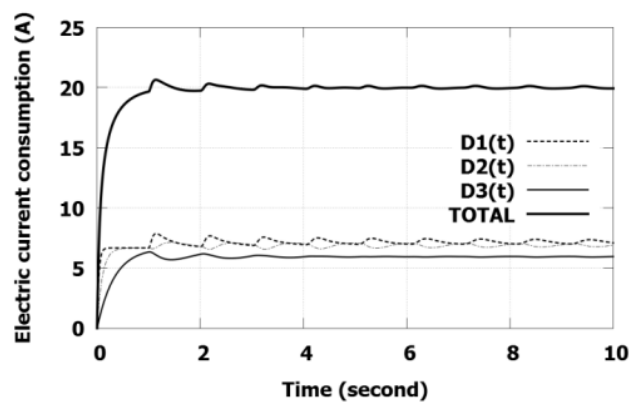

Fig. 6. Electric current consumption behavior of RBEC criteria with $a_{i}=30,10,3$ and $D_{i}^{\max }=12,7,5$ (for HA $i=1,2,3$ ).

Different delay values, $a_{i}$ and $D_{i}^{\max }$ were tried (not mentioned in this paper) and still similar behavior was observed. From 3rd and 4th rows of RBEC and EBEC having different values of the system parameter $a_{i}$ causes our

having same or different $D_{i}^{\max }$ values. With EBEC, overshoot happens faster than with the RBEC assignment criteria. On the other hand, RBEC (as compared to EBEC assignment criteria) takes less time to recover to stability after overshoot first happens.

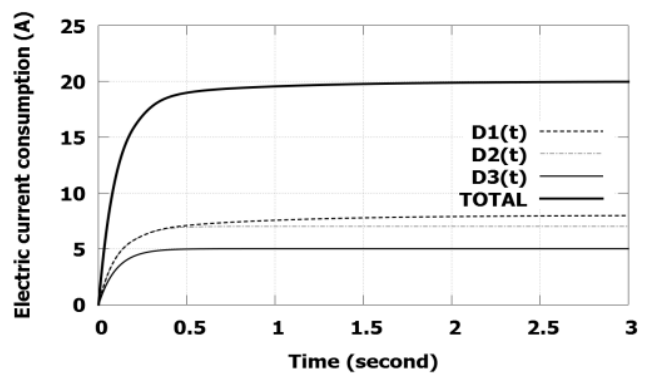

Fig. 7. Electric current consumption behavior of EBEC criteria with $a_{i}=10$ and $D_{i}^{\max }=12,7,5$ (for HA $i=1,2,3$ ). 


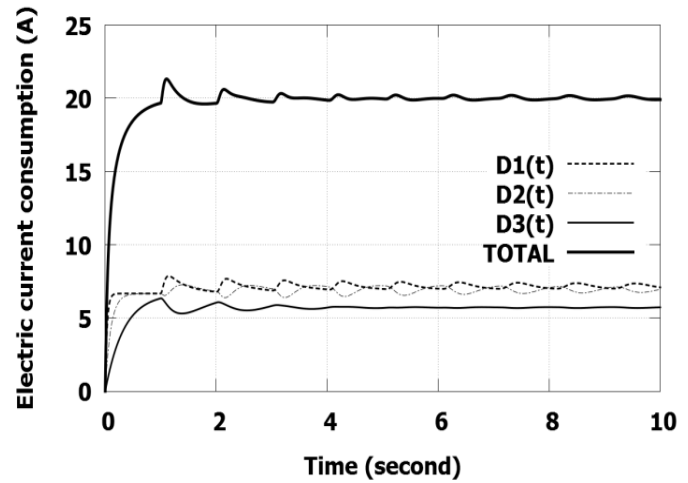

Fig. 8. Electric current consumption behavior of EBEC criteria with $a_{i}=30,10,3$ and $D_{i}^{\max }=12,7,5$ (for HA $i=1,2,3$ ).

Next we will show the second experiment we conducted to test the effect of turn ON timing on the final available electric current sharing. HAs having: $a_{i}=30,10,3$ and $D_{i}^{\max }=$ $10,7,5$ while the turns ON times are 0,2 , and 3.5 seconds.

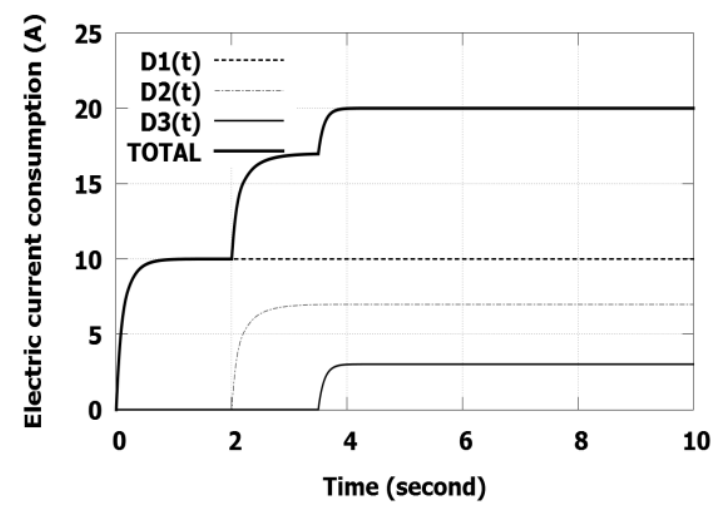

Fig. 9. Testing the effect of turn ON time with RBEC criteria.

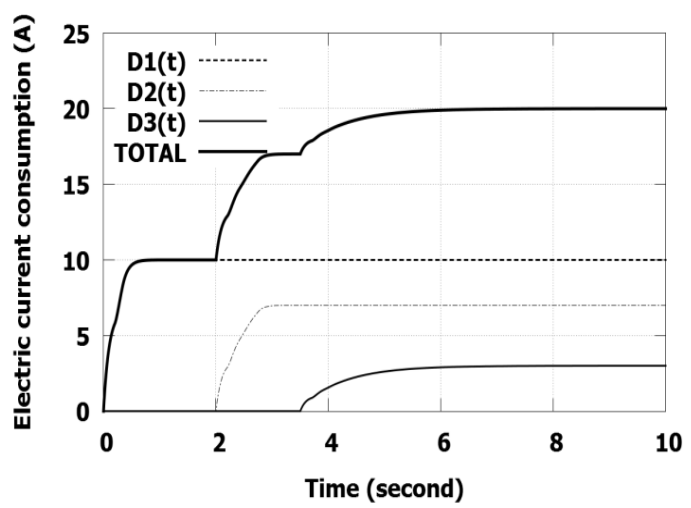

Fig. 10. Testing the effect of turn ON time with EBEC criteria.

In this experiment the HAs have different $a_{i}$ and $D_{i}^{\max }$ values from each other. In Fig. 9 and 10, RBEC and EBEC are shown respectively, both having the same values of system parameters. The HA which is turned ON first was found to use more available electric current while the last turned ON HA gets the smallest part of available electric current. We can also notice that RBEC response time in Fig.9 is a little different from that of EBEC in Fig. 10.

In our last experiment we will test the effect of different delay on the final available electric current sharing. In Fig. 11 and Fig. 12 the total consumed electric current is shown for different delay values for both of EBEC and RBEC assignment criteria respectively.

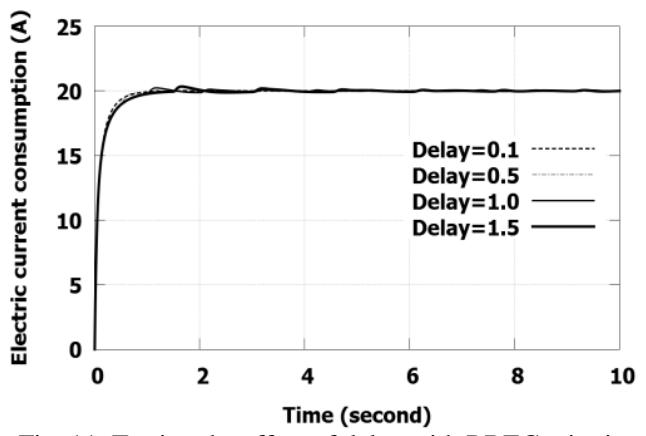

Fig. 11. Testing the effect of delay with RBEC criteria.

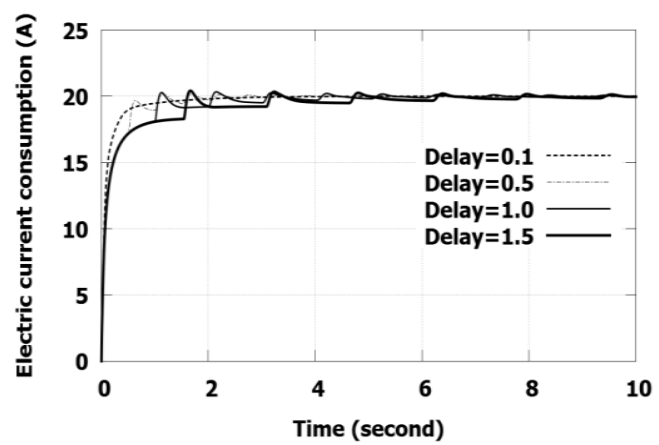

Fig. 12. Testing the effect of delay with EBEC criteria.

For the different delay values the other system parameters are kept as in the previous experiment. Only the turn on time is changed to the beginning of the simulation. We can see that with the EBEC assignment criteria the effect of delay causes more changes in the system total consumed electric current. While in the case of RBEC assignment criteria the changes are quite less. In both assignment criteria, the very small delay saves the system from having overshooting. As the delay starts growing to a more practical range both proposed criteria starts to exhibit overshooting at different times and with different overshooting duration and magnitudes.

\section{StABILITy ANALYSIS FOR PROPOSED SySTEM}

We carry out stability analysis for smart power management system which explains that indeed the stability is dependent on time delay $(\tau)$. Our focus is on the electric current consumption of the HAs. We can easily compare our proposed system equation (5) with linear time delay system equation having single delay given by:

$$
\dot{x}(t)=A_{0} \cdot x(t)+A_{1} \cdot x(t-\tau)
$$

where, $A_{0}=-a_{i}, A_{1}=a_{i}, x(t)=D(t), x(t-\tau)=D(t-$ $\tau)$ and $\dot{x}(t)=\dot{D}(t)$, the delayed term need to be analyzed for the stability of the system. For this purpose, we rewrite (5) with initial condition as follows:

$$
\begin{gathered}
\dot{D}(t)=A_{0} \cdot D(t)+A_{1} \cdot D(t-\tau) \\
D_{t}=\varnothing
\end{gathered}
$$

where $A_{0}$ and $A_{1}$ are given $n \times n$ real matrices. As the delayed term grows from zero, the system performance can deteriorate and system is in danger of losing stability. There is no loss of generality by rewriting the eq. (11) as, 


$$
\dot{D}(t)=\left(A_{0}+A_{1}\right) \cdot D(t)+A_{1} \cdot(D(t-\tau)-D(t))
$$

We consider the term $A_{1} \cdot(D(t-\tau)-D(t))$ as disturbance to the nominal stable system described as $\dot{D(t)}=\left(A_{0}+A_{1}\right) \cdot D(t)$. It is clear that the disturbance grows from zero as $\tau$ increases from zero.

This can be solved by model transformation in combination with delay independent stability for distributed delay problem, which helps us in deriving simple delay-dependent stability criteria. The delay-independent stability for distributed delay is given by,

$$
\dot{D}(t)=A_{0} \cdot D(t)+\int_{-\tau}^{0} A(\theta) \cdot(D(t+\theta) d \theta
$$

\section{A. Model Transformation}

We consider system eq. (11) with eq. (12), where the initial function is given as $\varnothing \in \mathcal{C}\left([-\tau, 0], \mathbb{R}^{n}\right)$, with the consideration that

$$
\begin{gathered}
D(t-\tau)=D(t)-\int_{-\tau}^{0} \dot{D}(t+\theta) d \theta \\
D(t-\tau)=D(t)-\int_{-\tau}^{0}\left[A_{0} \cdot D(t+\theta)+A_{1} \cdot D(t-\tau+\theta)\right] d \theta(16)
\end{gathered}
$$

for $t \geq \tau$, we can write eq. (11) as

$$
\begin{aligned}
& \dot{D}(t)=\left[A_{0}+A_{1}\right] \cdot D(t) \\
& +\int_{-\tau}^{0}\left[-A_{1} A_{0} \cdot D(t+\theta)-A_{1} A_{1} \cdot D(t-\tau+\theta)\right] d \theta
\end{aligned}
$$

with initial condition

$$
\begin{gathered}
D(\theta)=\psi(\theta),-\tau \leq \theta \leq \tau \\
\psi(\theta)=\left\{\begin{array}{cr}
\emptyset(\theta) & -\tau \leq \theta \leq 0 \\
\text { eq. (15) with eq. (10) } & 0<\theta \leq \tau
\end{array}\right.
\end{gathered}
$$

The system explains by (11) and (12) already exists in (17) and (18) without initial condition (19). As it is the time invariant system and for stability we can shift the initial time as

$$
\begin{gathered}
\dot{y}(t)=\overline{A_{0}} \cdot y(t)+\int_{-2 \tau}^{0} \bar{A}(\theta) \cdot y(t+\theta) d \theta \\
\left\{\begin{array}{c}
\overline{A_{0}}=A_{0}+A_{1} \\
\bar{A}(\theta)=-A_{1} A_{0}, \theta \in[-\tau, 0] \\
\bar{A}(\theta)=-A_{1} A_{1}, \theta \in[-2 \tau,-\tau]
\end{array}\right.
\end{gathered}
$$

with initial condition

$$
y(\theta)=\psi(\theta),-2 \tau \leq \theta \leq 0
$$

Let, $R(\theta)=\bar{A}(\theta) \cdot y(t+\theta)$. Now we transform our proposed system (11) and (12) by (20) and (21). Clearly the stability of the system (20) and (22) is the same as stability of the original system.

Theorem 1: [24] Power model described in eq. (11) is asymptotically stable if there is an existence of a real symmetric matrix $P>0$ such that:

$$
\left[\begin{array}{ccc}
M & -P A_{1} A_{0} & -P A_{1}^{2} \\
-A_{0}^{T} A_{1}^{2} P & -P & 0 \\
-\left(A_{1}^{2}\right)^{T} P & 0 & -P
\end{array}\right]<0
$$

where $M=\frac{1}{\tau}\left(P \hat{A}_{0}+P \hat{A}_{0}^{T}+2 P\right)$ and $\hat{A}_{0}=A_{0}+A_{1}$.

Proof: The proof is established by showing that $V=$ $D^{T} P D$ where $P$ is positive definite is a Lyapunov function. This in turn is shown by using eq. (24) and the existence of symmetric matrix by theorem 1 and the existence of symmetric matrix $R(\theta)$ in our transformed model that along with $P$ satisfies the conditions:

$$
\begin{gathered}
P \hat{A}_{0}+\hat{A}_{0}^{T} P+\int_{-2 \tau}^{0} R(\theta) d \theta<0 \\
{\left[\begin{array}{cc}
p P-R(\theta) & P \hat{A}(\theta) \\
-\hat{A}_{0} A_{1}^{2} P & -P
\end{array}\right]}
\end{gathered}
$$

With the help of theorem 1, the time delay system in (10) is stable provided by the time delay $\tau$ is such that theorem 1 is satisfied.

\section{NUMERICAL ANALYSIS}

In this section, we study the numerical evaluation of the stability theorem 1 discussed in previous section which guarantee that stability is dependent on time delay. At first, we consider the system equation in matrix notation for the value of system parameter $a_{i}$. Let $A_{0}=-a_{i}=-1, A_{1}=a_{i}=$ -1 (from matrix eq. 6) and $\hat{A}_{0}=A_{0}+A_{1}$. The total power available for the HAs is minimal (i.e. $P=1$ ) to observe the critical behavior of the system with $\tau=0.1$. The values of all system parameters are kept same for numerical evaluation except $\tau$. To prove the stability of proposed system, resulting value of theorem 1 should be negative. The evaluation of theorem 1 with above system parameters values and $\tau=0.1$ gives $-18<0$, which show that the system status is stable all the times. The evaluation criteria with $\tau=0.2$, the resulting value is $-8<0$, which shows that the system status is still stable. We repeat the evaluation process for $\tau=$ $0.3,0.4, \ldots, 0.9$ and at with an increase in the delay in information, the evaluation criteria show the resulting value less than zero, system is stable. At $\tau=1.0$ theorem 1 gives exactly zero which shows the marginal stability of the proposed system. In last, we test the theorem 1 with $\tau=1.1$ which gives $0.2>0$ shows that system status is unstable.

It can summarize that the theorem 1 can be used as tool to check the stability of the system globally. Moreover, this principle is also applicable to monitor the stability of the main breaker.

\section{CONCLUDING REMARKS}

In conclusion, the smart homes can be defined as a residence equipped with computing and information technology which anticipates and responds to the needs of the occupants, working to promote their comfort, convenience, security and entertainment. The efficiency, reliability and stability of smart homes can be significantly improved via 
our proposed PPC and SES. The use of SES was essential in the analysis of power consumption of HAs in home domain to get real time information.

In this paper, we studied the effect of SES that provides access to the power supply and demand in real time. In this paper we proposed an optimal system model for Smart Power Management System. In our proposed system design for smart homes, there are two key contributions; first information delay and second two suggested power assignment criteria for HAs. The simulation results show that the dependency of system parameter $a_{i}$ (HA response speed) in the system behavior such as stable/unstable, in other words overshoot/non-overshoot for both of the proposed power assignment criteria. We also notice the effect of different turn ON timing on total power consumption distribution for both of the proposed power assignment criteria.

For our future work, we will try to introduce priority of the HAs and analyze the factors affecting the system overshoot and stability.

\section{REFERENCES}

[1] F. Aldrich, "Smart homes: past, present and future," in Inside the Smart Homes, London: Springer-Verlag, 2003, pp. 17-40.

[2] L. Marusic, P. Skocir, A. Petric, and G. Jezic, "Home-in-palm a mobile service for remote control of household energy consumption," in Proc. 11th Int. Conf. on Telecommun., June 15-17, 2011, Austria, pp. 109-116.

[3] H. Lin, J. Chen, M. Jiang, and C. Huang, "Integration of GPRS and wireless LANs with multimedia applications," Adv. in Multimedia Inform. Process.-Lecture notes in Comput. Sci., vol. 2532, pp. 704-711, 2002.

[4] A. Busquet, G. Kardanas, J. Soler, and L. Dittmann, "Towards efficient energy management: defining HEMS, AMI and smart grid objectives," in Proc. The 10th Int. Conf. on Networks, 2011, pp. 249-263.

[5] M. J. Kofler, C. Reinisch, W. Kastner, and G. Kardaras, "An intelligent knowledge representation of smart home energy parameters," in Proc. Energy End-Use Efficiency Issues (EEE) World Renewable Energy Congr., May 2011, pp. 921-928.

[6] J. Han, C. Choi, W. Park, and I. Lee, "Green home energy management system through comparison of energy usage between the same kinds of home appliances," in Proc. IEEE 15th Int. Symp. On Consumer Electron., June 2011, pp. 1-4.

[7] D. Niyato, L. Xiao, and P. Wang, "Machine to machine communications for home energy management system in smart grid," IEEE Commun. Mag., vol. 49, no. 4, pp. 53-59, Apr. 2011.

[8] J. Barkans and D. Zalostiba, "New concept and solutions for prevention of power system blackout," in Proc. Int. Conf. on Develop. In Power System Protection, Apr. 2010, pp. 1-5.

[9] J. Barkans and D. Zalostiba, "Blackout prevention and power system self-restoration," in Proc. IEEE Int. Conf. on Comput. As Tool, Sept. 2007, pp. 1547-1554.

[10] Z. Shaobo and S. Zhanhui, "Challenges and opportunities in emergency management of electric power system blackout," in Proc. Int. Conf. on E-Product E-Service and E-Entertainment, Nov. 2010, pp. 1-4.

[11] J. Han, H. Lee, and K. R. Park, "Remote controllable and energy saving room architecture based on Zigbee communication," IEEE Trans. on Consumer Electron., vol. 55, no.1, pp. 264-268, Feb. 2009.

[12] S. Darby, "The effectiveness of feedback on energy consumption," Environmental Change Inst., Univ. of Oxford, Apr. 2006.

[13] M. Pipattanasomporn, M. Kuzlu, and S. Rahman, "An algorithm for intelligent home energy management and demand response analysis," IEEE Trans. On smart Grid, vol. 3, no. 4, pp. 2166-2173, Dec. 2012.

[14] K. Gill, S. H. yang, F. Yao, and X. Lu, "A zigbee- based home automation system," IEEE Trans. on Consumer Electron., vol. 55, no.2, pp. 422-430, May 2009.

[15] T. Facchinetti, E. Bini, and M. Bertonga, "Reducing the peak power through real-time scheduling techniques in cyber physical energy systems," presented at the 1st Int. Workshop on Energy Aware Design and Anal. of Cyber Physical Syst., 2010.

[16] A. R. Busquet, G. Kardaras, and V. B. I. J. Soler, "Reducing electricity demand peaks by scheduling home appliances usage," in Proc. Int Energy Conf., 2011, pp. 156-163.

[17] M. Kuzlu, M. Pipattanasomporn, and S. Rahman, "Hardware demonstration of a home energy management system for demand response applications," IEEE Trans. on Smart Grid, vol. 3, no. 4, pp. 1704-1711, Dec. 2012.

[18] M. Prymek and A. Horak, "Priority based smart household power control model," in Proc. IEEE Electrical Power and Energy Conf., 2012, pp. 337-343.

[19] G. Xiong, C. Chen, S. Kishore, and A. Yener, "Smart (In-home) power scheduling for demand response on the smart grid," in Proc. IEEE Innovative Smart Grid Technology, 2011, pp. 1-7.

[20] J. Han, C. Choi, and I. Lee, "More efficient home energy managemen system based on Zigbee communication and infrared remote controls," IEEE Trans. on Consumer Electron., vol. 57, issue. 1, pp. 85-89, 2011

[21] C. Len, Y. Bai, H. Chen, and C. Hung, "Home appliance energy monitoring and controlling based on power line communication," in Proc. Int. Conf. on Consumer Electron., 2009, pp. 1-2.

[22] T. Kato, K. Tamura, and T. Matsuyama, "Adaptive storage battery management based on the energy on demand protocol," IEEE Smart Grid Comm., pp. 43-48, 2012.

[23] S. Umer, M. Kaneko, Y. Tan, and A. O. Lim, "System design and analysis for maximum consuming power control in smart house," J. of Automation and Control Eng., vol. 2, no. 1, pp. 43-48, Sep. 2013.

[24] K. Gu, V. Kharitonov, and J. Chen, Stability of Time Delay System, Boston: Birkhuser, June 2003.

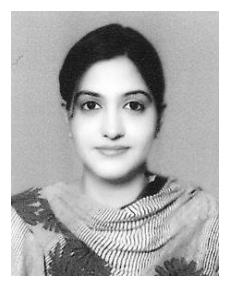

Saher Umer was born in Lahore, Pakistan. She received her BCS (Bachelor of Computer Science) from Allama Iqbal Open University, Islamabad, Pakistan in 2004. She also received her MSc-IT (Masters in Information Technology) from University of the Punjab (PUCIT), Lahore, Pakistan in 2007. She is currently a Ph.D. student in Graduate School of Information Science, Japan Advanced Institute of Science and Technology (JAIST). Her areas of research include Energy Management System for Smart Homes, Energy stability-aware schemes for Smart Homes, Renewable Energy contributions and Smart metering technologies for Smart Homes.

She also worked as a Visiting Lecturer at University of the Punjab and Education University, Lahore, Pakistan in 2010 and 2011 respectively.

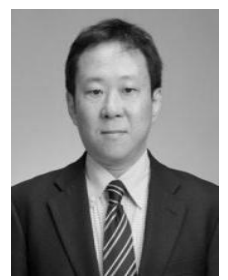

Yasou Tan received his Ph.D. from Tokyo Institute of Technology in 1993.Since then, he has been a member of the School of Information Science at Japan Advanced Institute of Science and Technology, and a professor since 1997. He is interested in ubiquitous computing systems, especially home network and smart home systems. He is the chairman of New Generation Network Promotion Forum Residentia ICT SWG, and Green Grid Platform at Home alliance, and a fellow of ECHONET consortium. He is a member of IEEE, ACM, IPSJ, IEICE, IEEJ, JSSST and JNNS.

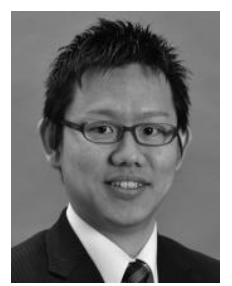

Azman Osman Lim received the B.Eng. (Hons) and M.Inf. Technology degrees from Universiti Malaysia Sarawak (UNIMAS), Malaysia in 1998 and 2000, respectively. He received the Ph.D. degree in communications and computer engineering from Kyoto University in 2005 . He was a visiting researcher at Fudan University in China for two months. During 2005-2009, he was an expert researcher at National Institute of Information and Communications Technology (NICT), Japan. Since 2009, he has been working at Japan Advanced Institute of Science and Technology (JAIST) as an associate professor. His research interests include multihop wireless networks, wireless sensor networks, home networks, wireless mesh networks, heterogeneous wireless networks, network coding, cyber-physical system. $\mathrm{He}$ is a member of IEEE, IEICE and IPSJ. 